\title{
USING CMOS SUB-MICRON TECHNOLOGY VLSI IMPLEMENTATION OF LOW POWER, High SPEED SRAM CELL AND DRAM CELL
}

\author{
Mr.Viplav A. Soliv ${ }^{1}$ Dr. Ajay A. Gurjar ${ }^{2}$ \\ ${ }^{1}$ Department of Electronics and Telecommunication \\ Sipna's college of Engineering \& Technology, Amravati. \\ viplove.soliverediffmail.com \\ ${ }^{2}$ Department of Electronics and Telecommunication \\ Sipna's college of Engineering \& Technology, Amravati. \\ prof_gurjar19280rediffmail.com
}

\begin{abstract}
Abstract This paper deals with the design and analysis of high speed Static Random Access Memory (SRAM) cell and Dynamic Random Access Memory (DRAM) cell to develop low power consumption. SRAM and DRAM cells have been the predominant technologies used to implement memory cells in computer systems, each one having its advantages and shortcomings. SRAM cells are faster and require no refresh since reads are not destructive. In contrast, DRAM cells provide higher density and minimal leakage energy. Here we use 12-transistor SRAM cell built from a simple static latch and tri state inverter. The reading action itself refreshes the content of memory. The SRAM access path is split into two portions: from address input to word line rise (the row decoder) and from word line rise to data output (the read data path). The decoder which constitutes the path from address input to the word line rise is implemented as a binary structure by implementing a multi-stage path. The key to low power operation in the SRAM data path is to reduce the signal swings on the high capacitance nodes like the bit lines and the data lines.
\end{abstract}

\section{KEYWORDS}

Keywords SRAM, DRAM, Low power, 12-T SRAM cell

\section{INTRODUCTION}

Static Random Access Memories (SRAM) and dynamic RAM (DRAM) have been the predominant technologies used to implement memory cells in computer systems. SRAM cells, typically implemented with six transistors (6T cells) have been usually designed for speed, while DRAM cells, implemented with only one capacitor and the corresponding pass transistor (1T1C cells) have been generally designed for density. Because of this reason, the former technology has been used to implement cache memories and the latter for main memory storage. Cache memories occupy an important percentage of the overall die area. A major drawback of these memories is the amount of dissipated static energy or leakage, which is proportional to the number of transistors used to implement these structures. However, although leakage currents are reduced, they still persist. In contrast, dynamic $1 \mathrm{~T} 1 \mathrm{C}$ cells avoid this drawback by design, since the power

DOI : $10.5121 /$ vlsic. 2011.2412 
supply is removed after accessing the memory cells. Typically, 1T1C DRAM cells were too slow to implement processor caches. However, technology advances have recently allowed embedding DRAM cells using CMOS technology. Despite technology advances, an important drawback of DRAM cells is that reads are destructive, that is, the capacitor loses its state when it is read. Table1 summarizes the main design characteristics of the discussed cells. In addition, capacitors lose their charge a long time, thus they must be recharged or refreshed. To refresh memory cells, extra refresh logic is required which in turn results not only in additional power consumption but also in availability overhead.

\begin{tabular}{|l|c|c|c|}
\hline Technology & SRAM (6T) & DRAM (1T1C) & eDRAM \\
\hline \hline Access time & fast & slow & slow \\
\hline Density & low & high & high \\
\hline Leakage & high & low & low \\
\hline Refresh & no & yes & yes \\
\hline Destructive reads & no & yes & yes \\
\hline
\end{tabular}

Table1: Memory Cell Characteristics

\subsection{An Introduction to SRAM}

The fundamental building block of a static RAM is the SRAM memory cell. The cell is activated by raising the word line and is read or written through the bit line. Fig (1) shows a 12-transistor SRAM cell built from a simple static latch and tri-state inverter. The cell has a single bit line. True and complementary read and write signals are used in place of a single word line. A representative layout in Fig (3) has an area of 46 x 75 X. The power and ground lines can be shared between mirrored adjacent cells, but the area is still limited by the wires and is undesirably large. However, the cell is easy to design because all nodes swing rail-to-rail and it is fast when used in small RAMs and register files. Fig (2) shows a 6-transistor (6T) SRAM commonly used in practice. Such a cell uses a single word line and both true and complementary bid lines. The complementary bit- line is often called bit or bit. The cell contains a pair of cross-coupled inverters and an access transistor for each bit line. True and complementary versions of the data are stored on the cross-coupled inverters. If the data is disturbed slightly, positive feedback around the loop will restore it to VDD or GND. The word line is asserted to read or write the cell.

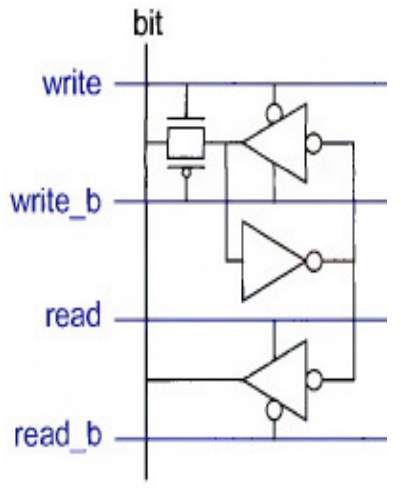

Fig 1: 12-transistor SRAM cell 
International Journal of VLSI design \& Communication Systems (VLSICS) Vol.2, No.4, December 2011

Two types of SRAM cells will be considered in this paper. (i) Conventional Twelve-transistor (12T) SRAM cell, as shown in Figure 1. (ii) Load less six-transistor (6T) SRAM Cell, as shown in Figure 2. They will be designed and analyzed in various configurations with respect to functionality, power dissipation, area occupancy, stability and access time. True and complementary versions of the data are stored on the cross-coupled inverters.

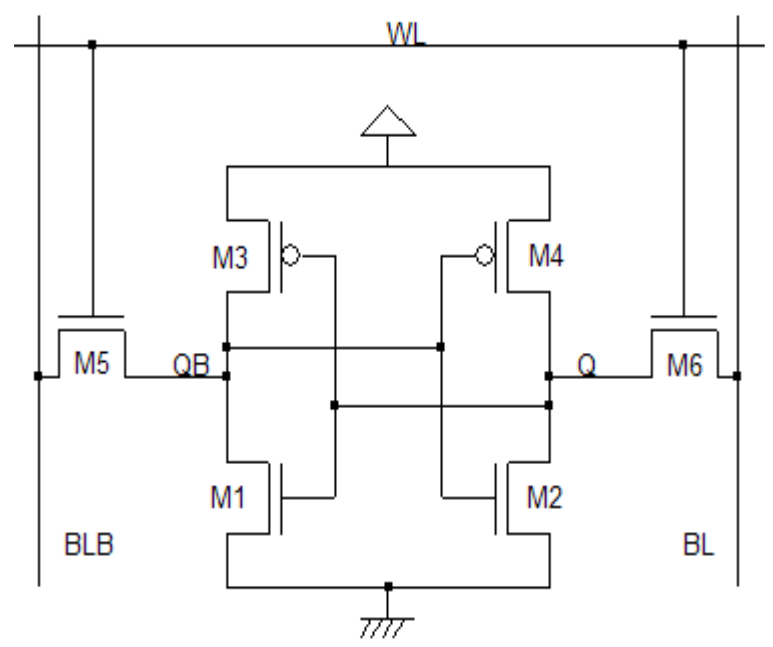

Fig 2: Conventional 6T SRAM Cell

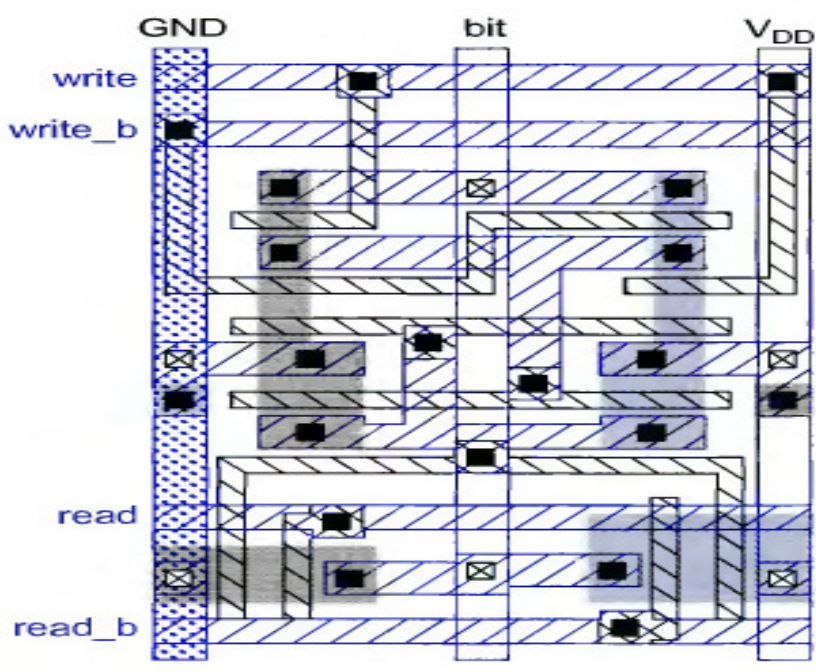

Fig 3: Representative layout of 46 x 75 X 
International Journal of VLSI design \& Communication Systems (VLSICS) Vol.2, No.4, December 2011

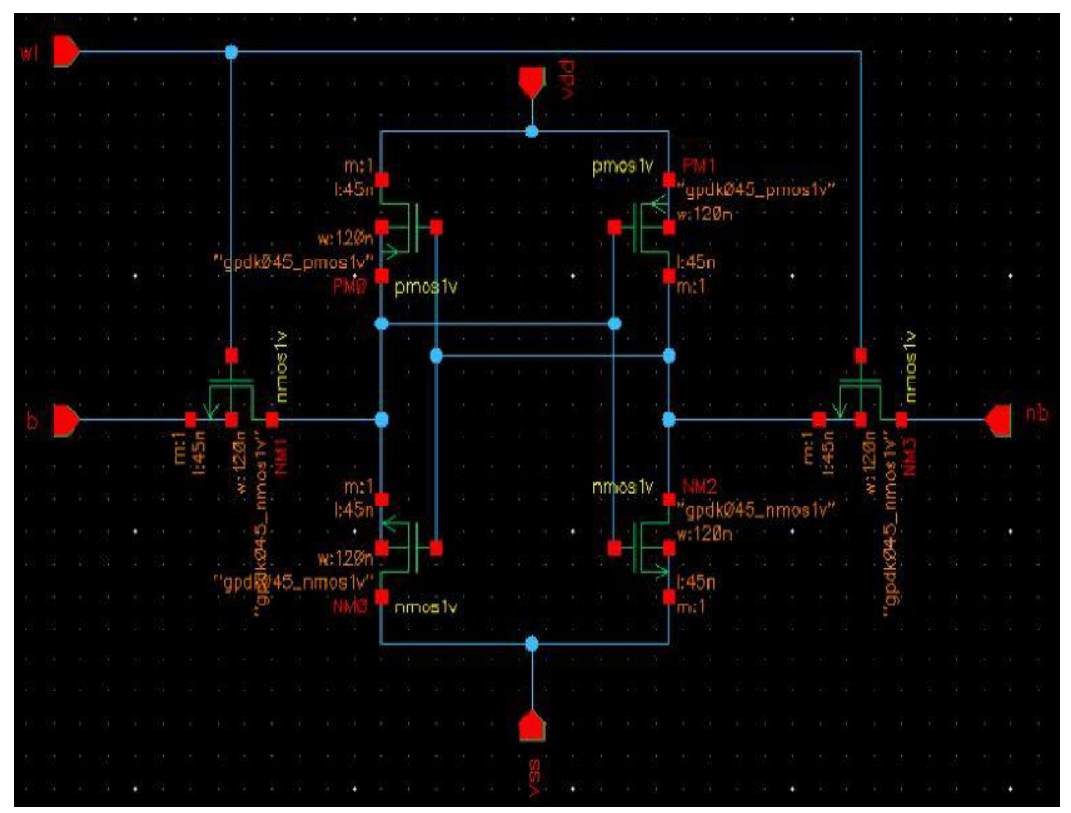

Fig 4: Schematic of SRAM cell

\subsection{An Introduction to DRAM}

Dynamic random access memory (DRAM) integrated circuits (ICs) have existed for more than twenty-five years. DRAMs evolved from the earliest 1-kilobit $(\mathrm{Kb})$ generation to the recent 1gigabit (GB) generation through advances in both semiconductor process and circuit design technology. Tremendous advances in process technology have dramatically reduced feature size, permitting ever higher levels of integration. These increases in integration have been accompanied by major improvements in component yield to ensure that overall process solutions remain cost-effective and competitive. Technology improvements, however, are not limited to semiconductor processing. Many of the advances in process technology have been accompanied or enabled by advances in circuit design technology. In most cases, advances in one have enabled advances in the other. Dynamic RAMs (DRAMs) store their contents as charge on a capacitor rather than in a feedback loop. Thus, the basic cell is substantially smaller than SRAM, but the cell must be periodically read and refreshed so that its contents do not leak away. Commercial DRAMs are built in specialized processes optimized for dense capacitor structures. They offer an order of magnitude greater density (bits/cm2) than high-performance SRAM built in a standard logic process, but they also have much higher latency. DRAM circuit design is a very specialized art. This section provides an overview of the general technique.

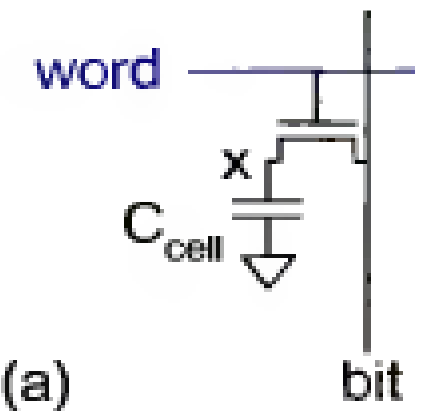


A 1-transistor (IT) dynamic RAM cell consists of a transistor and a capacitor, as shown in Figure (a). Like SRAM, the cell is accessed by asserting the word line to connect the capacitor to the bit line. On a read, the bit line is first pre charged to VDD/2. When the word line rises, the capacitor shares its charge with the bit line, causing a voltage change $\mathrm{A} V$ that can be sensed, as shown in Figure (b).

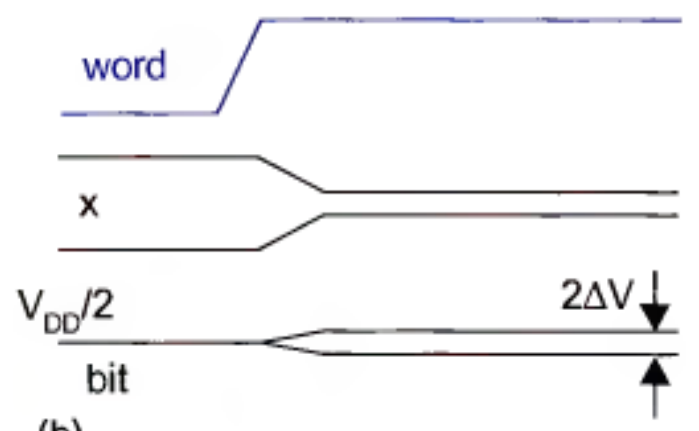

(b)

The read disturbs the cell contents at $\mathrm{x}$, so the cell must be rewritten after each read. On a write, the bit line is driven high or low and the voltage is forced onto the capacitor. Some DRAMs drive the word line to VDDP $=\mathrm{VDD}+\mathrm{Vt}$ to avoid a degraded level when writing a '1.' The DRAM capacitor must be as physically small as possible to achieve good density. However, the bit line is contacted to many DRAM cells and has a relatively large capacitance $\mathrm{C}$ bit. Therefore, the cell capacitance is typically much smaller than the bit line capacitance.

\section{MEMORY ARCHITECTURE}

The preferred organization for Random access memories is shown in Fig 5. This organization is random-access architecture which is an Asynchronous design. The name is derived from the fact that memory locations (addresses) can be accessed in random order at a fixed rate, independent of physical location, for reading or writing. The storage array, or core, is made up of simple cell circuits arranged to share connections in horizontal rows and vertical columns. The horizontal lines, which are driven only from outside the storage array, are called word lines, while the vertical lines, along which data flow into and out of cells, are called bit lines.

A cell is accessed for reading or writing by selecting its row and column. Each Cell can store 0 or 1. Memories may simultaneously select $4,8,16,32$, or 64 columns in one row depending on the application. The row and column (or groups of columns) to be selected are determined by decoding binary address information. In this design, the number of rows and columns, both are equal to 64 for $4 \mathrm{Mb}$ memory cut. Using two such memory cuts, a 8Mb SRAM memory is designed. 
International Journal of VLSI design \& Communication Systems (VLSICS) Vol.2, No.4, December 2011

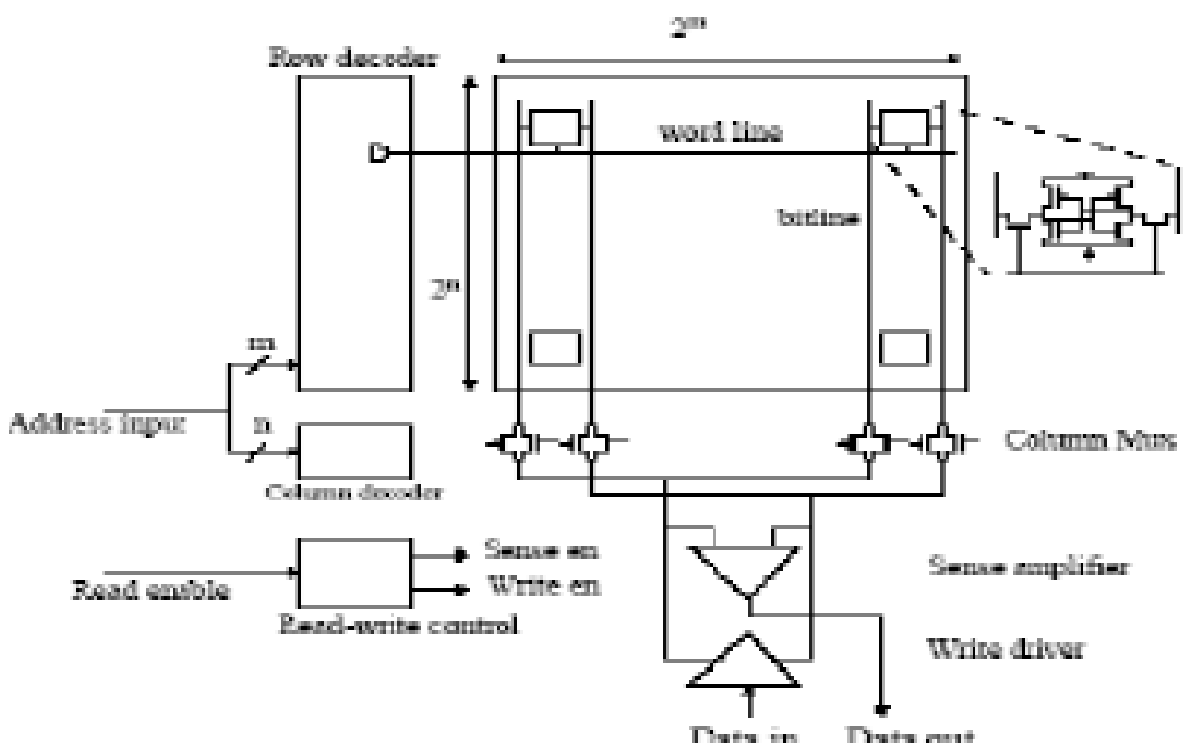

Fig 5: SRAM Memory Architecture

\section{SENSE AMPLIFIER}

The sense amplifiers have to amplify the data which is present on the bit lines during the read operation. The memory cells are small in size, and hence cannot the discharge the bit lines fast enough. Also, the bit lines continue to slew till a large differential voltage is formed between them. This causes significant power dissipation since the bit lines have large capacitances.

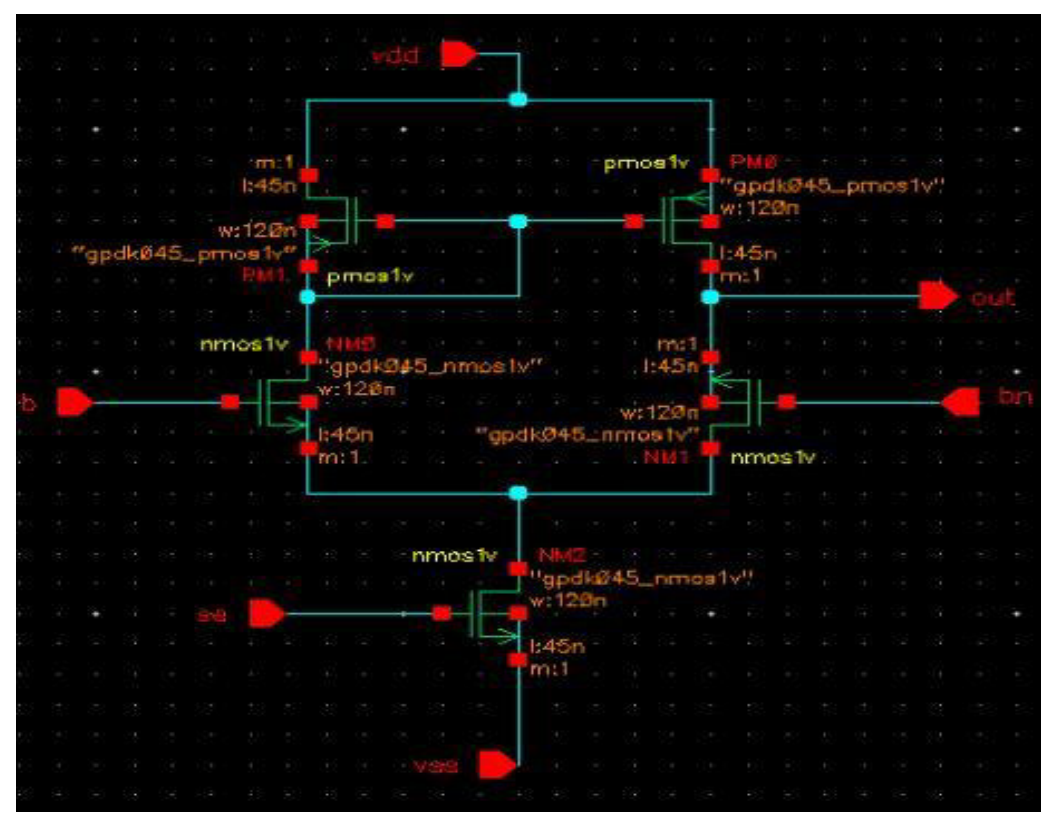

Fig 6: Schematic of sense amplifier 
Hence, by limiting the word line pulse width we can control the amount of charge pulled down by the bit lines and hence limit power dissipation. The schematic and symbol are as shown below. It consists of two cross coupled gain stages which are enabled by the sense clock signal. The cross coupled stage ensures a full amplification of the input signal. This type of amplifier consumes least amount of power, however they can potentially be slower since some timing margin is needed for the generation of the sense clock signal. If the sense amplifiers enabled before sufficient differential voltage is formed, it could lead to a wrong output. Thus, the timing of the sense clock signal needs to be such that the sense amplifier can operate over various process corners and temperature ranges.

In this sense amplifier Bit lines have many cells attached. If we take example of that Ex: 32-kbit SRAM has 256 rows x 128 cols. On each bit line 128 cells are present.

Sense amplifiers are triggered on small voltage swing (reduce $\Delta \mathrm{V}$ ).Even with shared diffusion contacts, $64 \mathrm{C}$ of diffusion capacitance are there. Discharged slowly through small transistors.

\section{SIMULATION ENVIRONMENT AND RESULTS}

The following configuration of SRAM arrays were designed and analyzed using the conventional 6T SRAM Cell: (a) $1 * 1$ (b) $16 * 16$ (c) $32 * 32$. The various configurations were simulated using HSPICE using the Nominal Predictive Technology Model (PTM) in 130nm, 90nm and 65nm CMOS technologies. The functionality of $1 * 16 \mathrm{~T}$ SRAM cell is shown in Figure. 7 The For 1Kbit $(32 * 32)$ configuration along with the relevant input control signals, only the signals for three input data bits (0th, 16th and 31st), three output data bits (0th,16th and 31st), and the corresponding storage nodes of the appropriate cell is presented.

Cadence simulation of transient analysis and DC analysis gave good results. The results are shown in the timing diagram. The noise margins are very good and the output is stable.

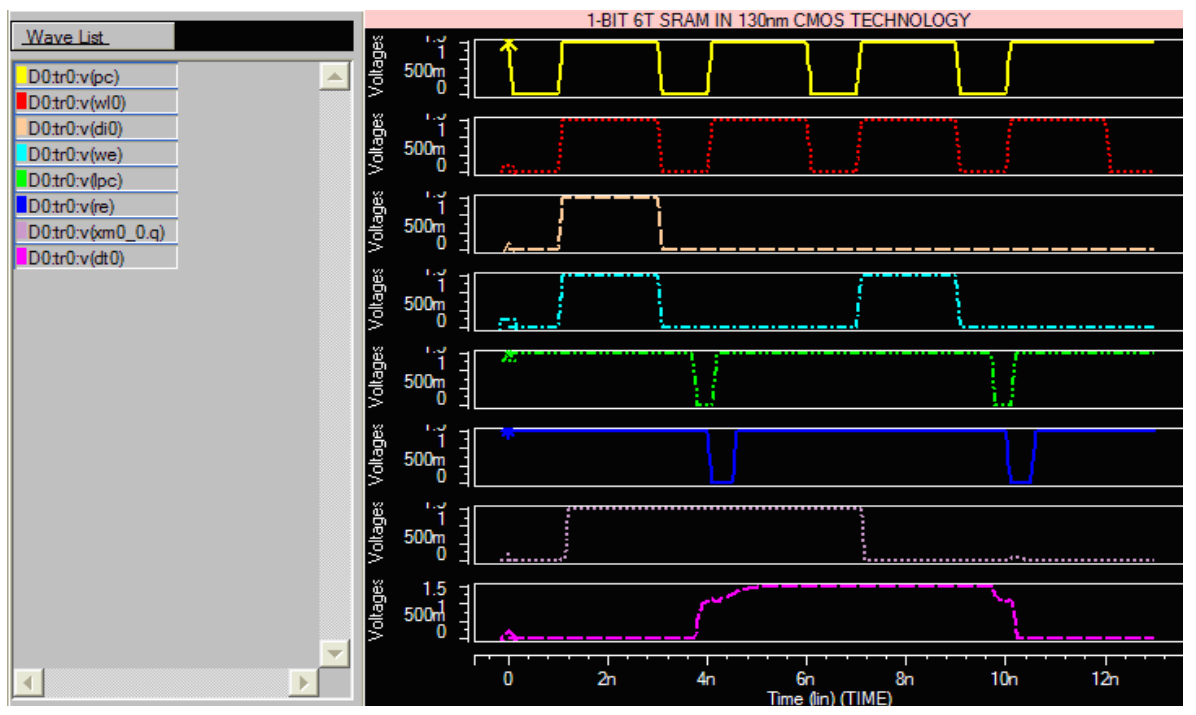

(a) 
International Journal of VLSI design \& Communication Systems (VLSICS) Vol.2, No.4, December 2011
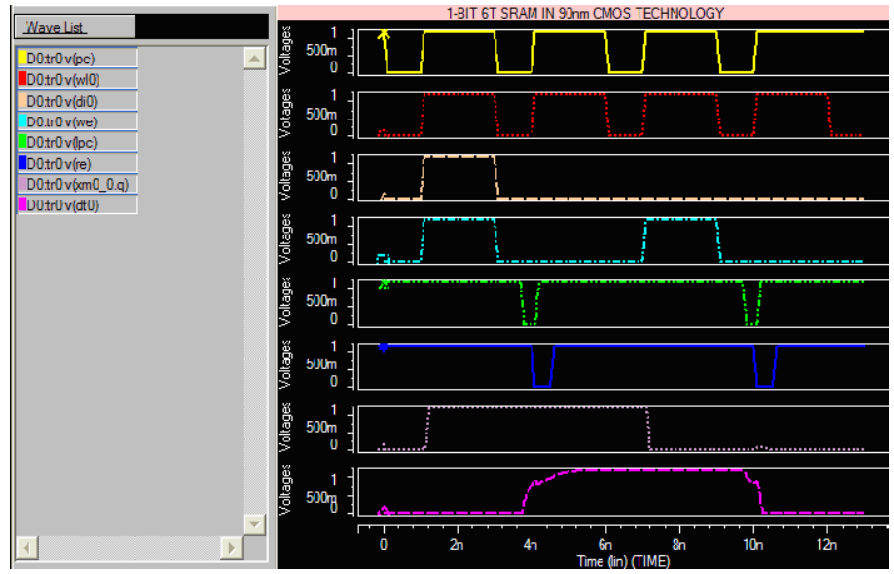

(b

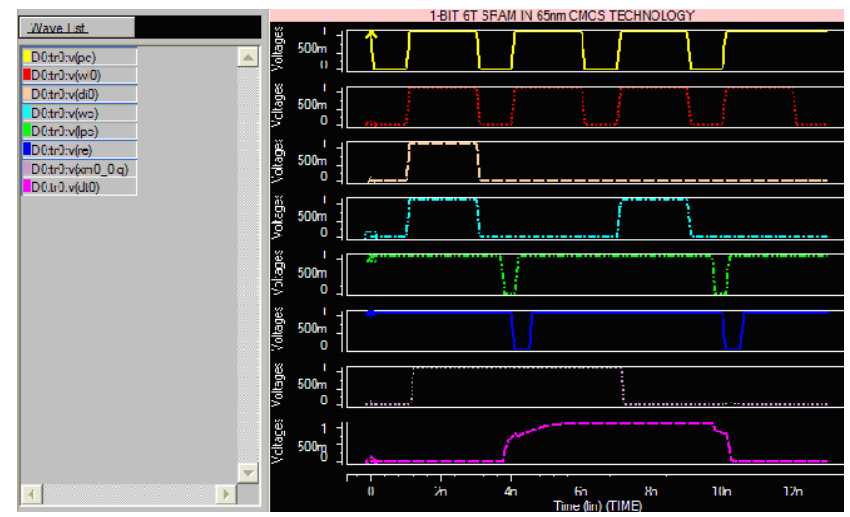

(c)

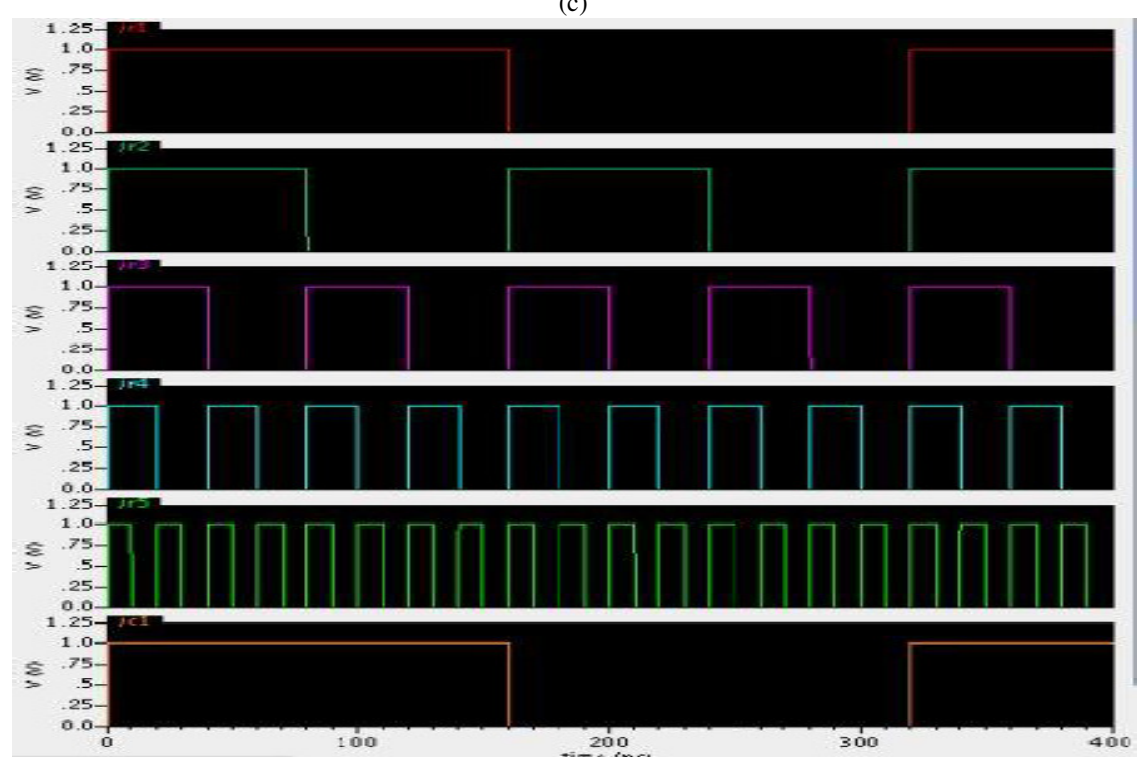

Fig 8: Timing Diagram 
International Journal of VLSI design \& Communication Systems (VLSICS) Vol.2, No.4, December 2011

\section{DESIGN FLOW}

Cadence design Systems is electronic design automation software and engineering Services Company that offers various types of design and verification tasks that include:

Virtuoso Platform - Tools for designing full-custom integrated circuits, includes schematic entry, behavioral modeling (Verilog-AMS), circuit simulation, full custom layout, physical verification, extraction and back-annotation.

Encounter Platform - Tools for creation of digital integrated circuits. This includes floor planning, synthesis, test, and place and route.

Incisive Platform - Tools for simulation and functional verification of RTL including Verilog, VHDL and System C based models. Includes formal verification, formal equivalence checking, hardware acceleration, and emulation.

The proposed work is done in Virtuoso platform using gpdk45 nm technology. The flow of design is as shown below.

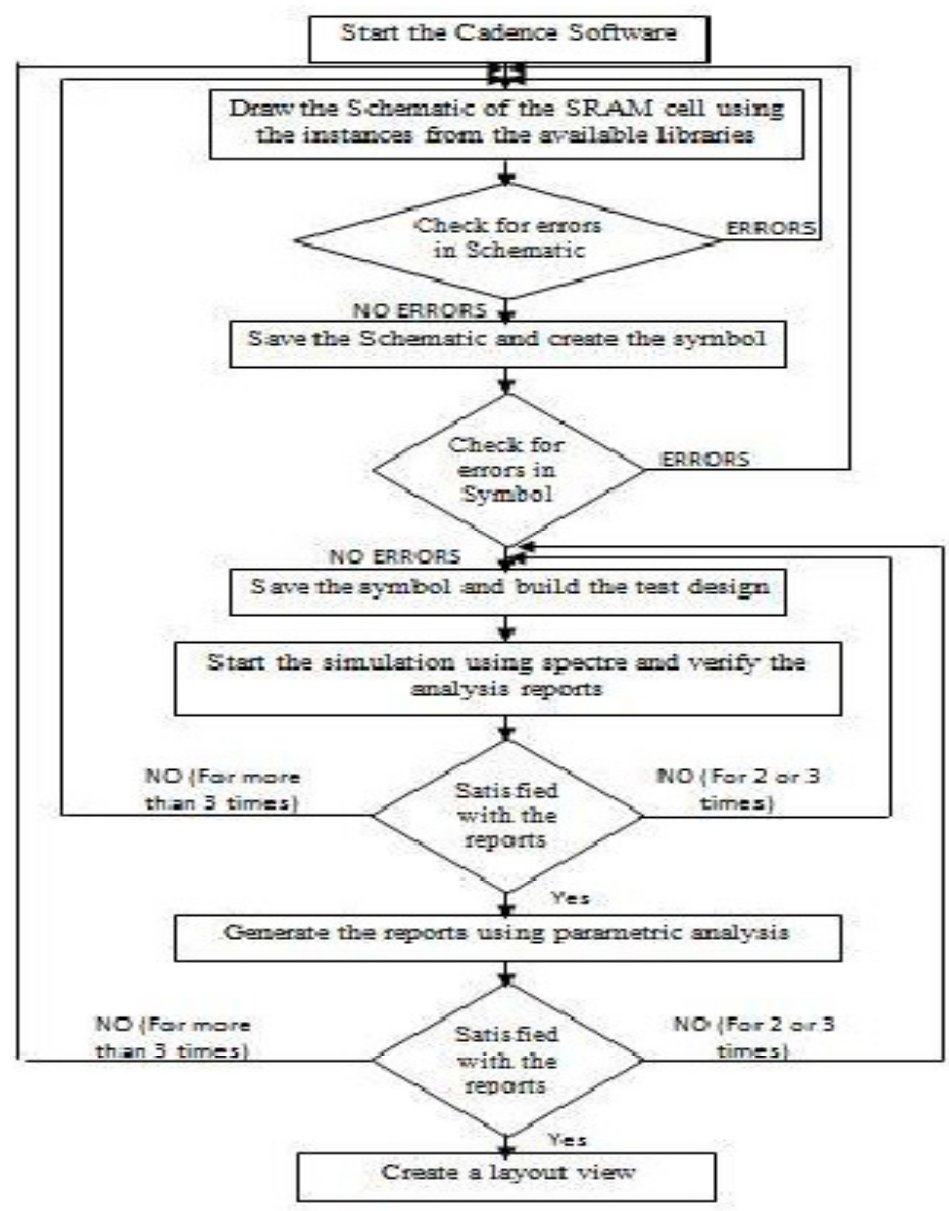

Fig 9: Design Flow 


\section{Conclusion}

The New Load less 6T-SRAM cell is designed and analyzed in deep submicron $(130 \mathrm{~nm}, 90 \mathrm{~nm}$ and $65 \mathrm{~nm}$ ) CMOS technologies, which establish the technology independence of the New Load less 4T SRAM cell and its consistent performance with respect to Conventional 6T SRAM cell in deep sub-micron regime. The New Load less 6T SRAM array consumes low power with low area. The most significant feature of this new load less 4T SRAM Cell is that there is no need to modify any of the fabrication process. Thus it can be used for on-chip caches in embedded microprocessors, high density SRAMs embedded in any logic devices, as well as for stand-alone SRAM applications.

This paper presents the design of SRAM array in $45 \mathrm{~nm}$ having very low power consumption. The low power design of SRAM is investigated and 6T SRAM architecture is chosen for memory bit cell and an array is designed with that bit cell. Transient and parametric analyses were carried out in the simulation process and the power consumption is estimated. As stated earlier, the power consumption can further be reduced by partitioning the array and by using DWL scheme.

\section{REFERENCES}

[1] M. Yamaoka, N. Maeda, Y. Shinozaki, Y. Shimazaki, K. Nii, S. Shimada, K. Yanagisawa, and T. Kawahara, "90-nm process-variation adaptive embedded SRAM modules with power-line-floating write technique,” IEEE J. Solid-State Circuits, vol. 41, no. 3, pp. 705-711,Mar. 2006.

[2] E. Seevinck et al., "Static-noise margin analysis of MOS SRAM cells," IEEE J. Solid-State Circuits, vol. SC-22, no. 2, pp. 748-754, May 1987.

[3] Sreerama Reddy G.M, P.Chandrashekara Reddy "Design and VLSI Implementation of 8 Mb Low Power SRAM in 90nm" European Journal of Scientific Research ISSN 1450-216X Vol.26 No.2 (2009), pp.305-314.

[4] Anne-Johan Annema, Member, IEEE, Bram Nauta, Senior Member, IEEE, Ronald van Langevelde, Member, IEEE and Hans Tuinhout "Analog Circuits in Ultra-Deep-Submicron CMOS" IEEE JOURNAL OF SOLID-STATE CIRCUITS, VOL. 40, NO. 1, JANUARY 2005.

[5] "Dynamic RAM" ELEC 464 : MICROCOMPUTER SYSTEM DESIGN 1996/97 WINTER SESSION TERM 1

[6] John Poulton, "An Embedded DRAM for CMOS ASICs"

[7] Alejandro Valero, Julio Sahuquillo, Salvador Petit, Vicente Lorente,Ramon Canal, Pedro López, José Duato "An Hybrid eDRAM/SRAM Macrocell to Implement First-Level Data Caches"

[8] James S. Caravella, “A Low Voltage SRAM for EmbeddedApplications”, IEEE Journal of Solid-State Circuits, vol. 32, no. 3, pp. 428 - 432, March 1997.

[9] Delaluz, V., Sivasubramaniam, A., Kendemir, M., Vijaykrishnan N., and Irwin, M.,Scheduler-Based DRAM Energy Management, Design Automation Con-ference, 2002.

[10] M. Yamaoka, K. Osada, R. Tsuchiya, M. Horiuchi, S. Kimura, and T. Kawahara, "Low power SRAM menu for SOC application using Yin- Yang-feedback memory cell technology," in Symp. VLSI Technology 2004 Dig. Tech. Papers, Jun. 2004, pp. 288-291.

[11] Neeraj Kr. Shukla, R.K.Singh, Manisha Pattanaik "Design and Analysis of a Novel Low-Power SRAM Bit-Cell Structure at Deep-Sub-Micron CMOS Technology for Mobile Multimedia 
International Journal of VLSI design \& Communication Systems (VLSICS) Vol.2, No.4, December 2011

Applications" (IJACSA) International Journal of Advanced Computer Science and Applications, Vol. 2, No. 5, 2011

[12] Brent Keeth "A NOVEL ARCHITECTURE FOR ADVANCED HIGH DENSITY DYNAMIC RANDOM ACCESS MEMORIES” May 1996

[13] Xiaoyao Liang, Ramon Canal, Gu-Yeon Wei David Brooks "REPLACING 6T SRAMS WITH 3T1D DRAMS IN THE L1 DATA CACHE TO COMBAT PROCESS VARIABILITY” IEEE 02721732/08/\$20.0 G 2008 JANUARY-FEBRUARY 2008.

[14] Paridhi Athe, S. Dasgupta “A Comparative Study of 6T, 8T and 9T Decanano SRAM cell” 2009 IEEE Symposium on Industrial Electronics and Applications (ISIEA 2009), October 4-6, 2009, Kuala Lumpur, Malaysia.

[15] Pramod Kolar, Member, IEEE, Eric Karl, Member, IEEE, Uddalak Bhattacharya, Member, IEEE, Fatih Hamzaoglu, Member, IEEE, Henry Nho, Yong-Gee Ng, Yih Wang, Member, IEEE, and Kevin Zhang, Senior Member, IEEE “A 32 nm High-k Metal Gate SRAM With Adaptive Dynamic Stability Enhancement for Low-Voltage Operation” IEEE JOURNAL OF SOLID-STATE CIRCUITS, VOL. 46, NO. 1, JANUARY 2011.

[16] Sandeep R, Narayan T Deshpande, A R Aswatha, "Design and Analysis of a New Loadless 4T SRAM Cell in Deep Submicron CMOS Technologies" Second International Conference on Emerging Trends in Engineering and Technology, ICETET-09.

[17] James S. Caravella, “A Low Voltage SRAM for EmbeddedApplications”, IEEE Journal of Solid-State Circuits, vol. 32, no. 3,pp. 428 - 432, March 1997.

[18] Jinshen Yang and Li Chen, "A New loadless 4-transistor SRAM cell with a $0.18 \mu$ m CMOS technology", Electrical and Computer Engineering, CCECE Canadian Conference, pp. 538 - 541, April 2007.

[19] P.Y. Chee, P.C. Liu, L. Siek, "High-speed hybrid current-mode sense amplifier for CMOS SRAMs", ELECTRONICS LETTERS, vol. 28, no. 9, April 1992.

[20] Jinn-Shyan Wang, Hong-Yu Lee, “A new current-mode sense amplifier for low- voltage low-power SRAM design”, Eleventh Annual IEEE International Proceeding of ASIC, pp.163-167, Sep. 1998.

\section{Authors}

Mr. Viplav A. Soliv is currently working as a lecturer in Electronics and Telecommunication Engineering Department, Sipna College of Engineering, Amravati (India) since 2010. He is also persuing his M.E. in Digital Electronics from the same institute. His areas of interest are Digital System Design, VLSI Design.

viplove.soliv@ rediffmail.com

Dr. Ajay A. Gurjar is currently working as a Professor in Electronics and Telecommunication Engineering Department, Sipna College of Engineering; Amravati (India).He also completed his Ph.D in signal processing. His areas of interest are Digital System Design, VLSI Design, Image Processing, and Signal processing.

prof_gurjar1928@rediffmail.com
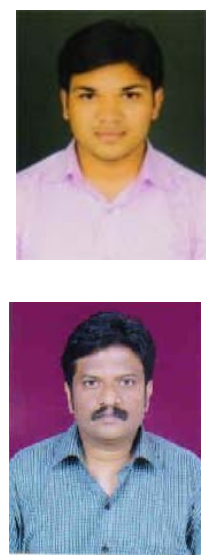